\title{
Medición de la calidad de vida por medio del Dermatology Life Quality Index en pacientes con psoriasis: una revisión sistemática
}

Assessing quality of life through the Dermatology Life Quality Index in patients with psoriasis: a systematic review

\author{
Jaime Ordóñez ${ }^{1}$, Árlex Palacios², Ángela Londoño ${ }^{3}$, Sol Jiménez ${ }^{4}$ \\ 1. Investigador de HEMOgroup, Medellín, Colombia. \\ 2. Investigador, Centro de Evaluación de Tecnologías en Salud de la Universidad CES-CETES, Medellín, Colombia \\ 3. Coordinadora, Grupo de Investigación de Dermatología, Escuela de Ciencias de la Salud, Universidad Pontificia Bolivariana, Medellín, Colombia \\ 4. Directora, Departamento de Dermatología, Facultad de Medicina, Universidad CES, Medellín, Colombia
}

\section{Resumen}

Objetivo. Evaluar la calidad de vida relacionada con la salud en pacientes con psoriasis, por medio del Dermatology Life Quality Index (DLQI).

Metodología. Revisión sistemática de estudios sobre calidad de vida relacionada con la salud en pacientes con psoriasis y en que se haya utilizado el DLQI. La búsqueda incluyó estudios publicados entre el 1994 y el 2012. Se excluyeron los estudios que evaluaran la calidad de vida relacionada con los tratamientos farmacológicos.

Resultados. En ocho estudios se cumplieron los criterios de inclusión y exclusión. Hubo una pérdida de la calidad de vida en los pacientes con psoriasis. El DLQI fue reportado en dos estudios con promedios de 6,4 y 10,8; la duración de la enfermedad varió entre 17,6 y 28,9 años.

Conclusión. La psoriasis afecta la calidad de vida de los pacientes que padecen la enfermedad, tanto en su forma leve como moderada, y este deterioro es superior al encontrado en enfermedades como el acné o el vitiligo.

PALABRAS CLAVE: psoriasis, calidad de vida, reproducibilidad de resultados, impacto de la evaluación de resultados.

\section{Summary}

Objective: To evaluate quality of life related to health in patients with psoriasis, through the Dermatology Life Quality Index (DLQI).

Methods: A systematic review about quality of life related to health in patients with psoriasis and has been used the DLQI. Search included studies published between 1994 and 2012. Studies that evaluated quality of life related to drug treatments were excluded.

Results: Eight studies met the inclusion and exclusion criteria. There was a loss of quality of life in patients with psoriasis. DLQI was reported in two studies with 6.4 and 10.8 averages; disease length ranged between 17.6 and 28.9 years. Conclusion: Psoriasis affects quality of life of patients with the disease, from mild to moderate forms, and this deterioration is greater than those found in patients with acne or vitiligo.

KEY WORDS: Psoriasis, quality of life, reproducibility of results, health impact assessment.
Correspondencia:

Jaime Ordóñez

Email:

gerencia@hemogroup.com.co

Recibido 3 de junio de 2013.

Aceptado: 10 de septiembre de 2013.

Conflictos de intereses: Esta investigación fue desarrollada por el Centro de Evaluación de Tecnologías en Salud de la Universidad CES y fue financiado por Pfizer. Los investigadores declaran su total independencia del patrocinador y son los únicos responsables de los métodos, conceptos y resultados presentados en este estudio. Pfizer no tuvo ningún papel en el desarrollo de esta investigación. 


\section{Introducción}

La psoriasis es una enfermedad inflamatoria crónica de la piel que afecta del 2 al $3 \%$ de la población mundial, y se caracteriza por la hiperproliferación y diferenciación incompleta de la capa epidérmica ${ }^{1-3}$. De Arruda ${ }^{4}$ observó que $79 \%$ de los pacientes con psoriasis habían manifestado que la enfermedad tenía un impacto negativo en su calidad de vida. Asimismo, algunos experimentaron deterioro en sus actividades diarias, laborales e interpersonales, y discriminación; además, $10 \%$ de los pacientes entre 18 y 34 años contempló la posibilidad del suicidio.

Existen diferentes instrumentos para medir la calidad de vida relacionada con la salud en la psoriasis, que van desde cuestionarios elaborados por expertos hasta escalas validadas y aplicadas en diferentes países como el Reino Unido, los Estados Unidos y Alemania, entre otros $^{5-14}$. De igual forma, existe una variedad de cuestionarios que miden la calidad de vida de forma específica en pacientes con psoriasis, sin que existan criterios homogéneos sobre cuándo utilizar cada uno de ellos ${ }^{7-9,15,16}$.

Entre los múltiples instrumentos utilizados para medir la calidad vida relacionada con la salud, el más utilizado es el Dermatology Life Quality Index (DLQI), especialmente en personas de 16 años o más. Se trata de un cuestionario autoadministrado, que no requiere información detallada para diligenciarlo, se completa en dos minutos ${ }^{17-19} \mathrm{y}$ valora la calidad de vida durante la última semana. Fue desarrollado en 1994 por Finlay, et $a l .,{ }^{17} \mathrm{y}$ fue el primer instrumento en medir de forma específica la calidad de vida en dermatología.

El DLQI evalúa seis dimensiones: síntomas y sentimientos sobre la enfermedad, actividades diarias, ocio, trabajo y estudio, relaciones personales y tratamiento. El instrumento consta de diez preguntas, cuyas puntaciones son interpretadas como: 0-1, calidad de vida no afectada; 2-5, poco afectada; 6-10, moderadamente afectada; 11-20, muy afectada; y 21-30, extremadamente afectada. Se ha utilizado en más de 32 enfermedades dermatológicas en 33 países y se ha traducido a 85 idiomas. Ha sido descrito en más de 800 publicaciones, especialmente en ensayos clínicos, y es el instrumento más usado para medir la calidad de vida en pacientes con lesiones dermatológicas ${ }^{20}$.

La existencia de una variedad de reportes en los que se utilizan diferentes escalas de medición de la calidad de vida ${ }^{21-24}$, lleva al fraccionamiento y confusión en la interpretación de la información, debido a que los resultados son diferentes según el instrumento utilizado y no permiten apreciar de forma general cómo afecta la psoriasis la calidad de vida.

El objetivo de esta revisión sistemática fue determinar el impacto de la psoriasis leve, moderada y grave, sobre la calidad de vida, medida a través del DLQI, reportando la medida explícita de los estudios y el Psoriasis Assessment and Severity Index (PASI) como resultado secundario, cuando este se reporta.

\section{Metodología}

Se hizo una búsqueda sistemática en Pubmed, Cochrane Library, Embase y CINAHL, sobre la calidad de vida relacionada con la salud en pacientes con psoriasis leve, moderada y grave. Se incluyeron estudios clínicos experimentales y observacionales que evaluaran la calidad de vida en pacientes adultos mediante el DLQI, y cuando este índice estuviese validado en el país del estudio. La búsqueda incluyó estudios en inglés publicados desde 1994 hasta diciembre de 2012. Se utilizaron los mismos términos de búsqueda utilizados por Basra, et al., ${ }^{25} \mathrm{y}$ se complementó la búsqueda con los siguientes términos MeSH: psoriasis AND quality of life, impact of psoriasis quality of life, psoriasis AND Dermatology Life Quality Index. El análisis de los resultados fue cualitativo, para determinar la validez interna de los resultados.

Se excluyeron artículos que comparaban diferentes tipos de tratamiento en términos de mejoría de la calidad de vida antes de la administración de medicamentos y después de ella, artículos cuyo objetivo fuese la validación del DLQI, así como aquellos que evaluaban la calidad de vida de variantes de la enfermedad, tales como artritis psoriásica y psoriasis de las uñas.

Los textos completos de los artículos fueron evaluados de forma independiente por dos médicos epidemiólogos, para determinar su validez interna, así como el cumplimiento de los criterios de inclusión y exclusión; los desacuerdos fueron resueltos por una dermatóloga. No se hizo cegamiento de los revisores respecto al autor y revista, porque el número de artículos obtenidos fue muy alto y hacerlo no generaba ningún valor agregado, toda vez que existen otros métodos de identificar artículos en internet sin considerar el autor y la revista, perdiéndose el objetivo de dicho cegamiento. El resultado primario fue la calidad de vida relacionada con la salud medida mediante el DLQI de manera auto-administrada o por el médico, y se reporta como resultado secundario el PASI.

\section{Resultados}

Con la combinación de los términos "psoriasis AND quality of life" se identificaron 1.187 artículos; con el término "impact of psoriasis in quality of life", 218; y con los términos "psoriasis AND Dermatology Life Quality Index", 402. Una vez leídos los títulos y resúmenes de cada artículo, se identificaron 87 artículos potenciales, de los 


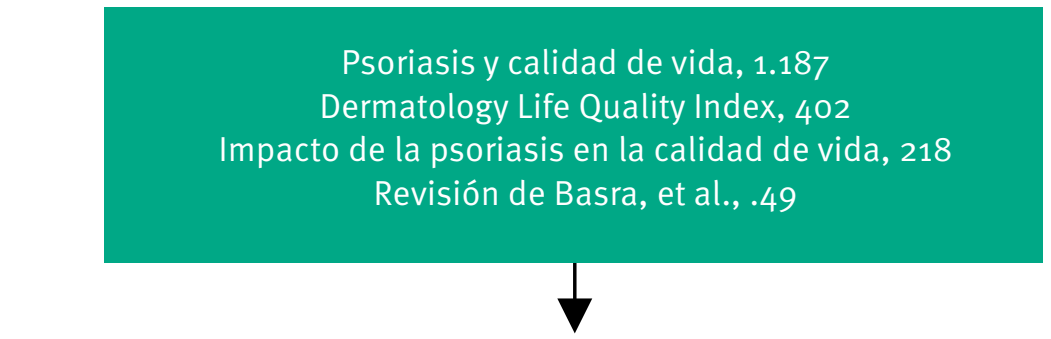

Artículos obtenidos después de la revisión de títulos y resúmenes, 87

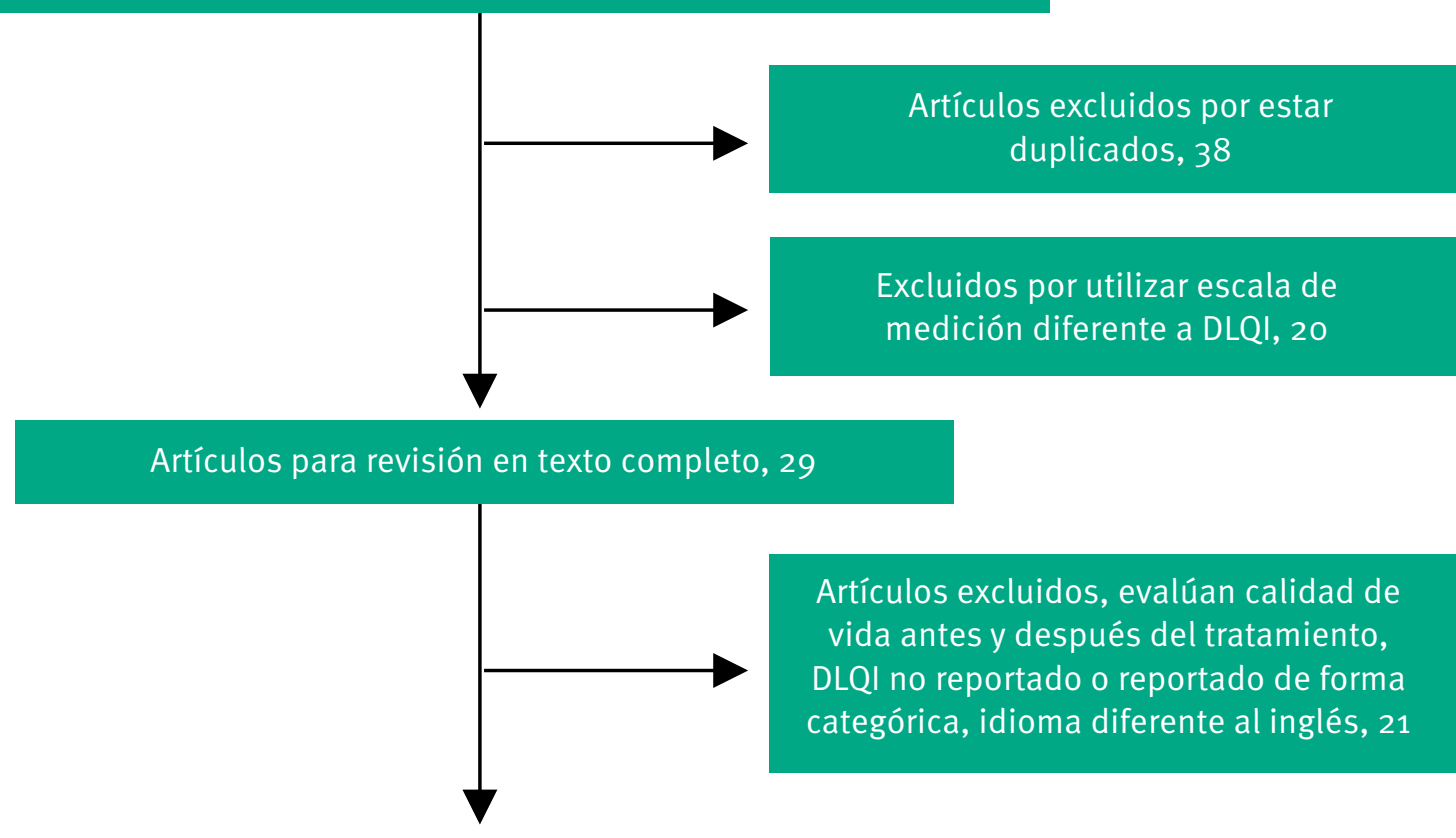

Artículos incluidos en la revisión sistemática, 8

FigURA 1. Diagrama de flujo de los hallazgos de la estrategia de búsqueda para identificar estudios que hayan evaluado la calidad de vida en pacientes con psoriasis por medio del DLQI.

cuales se excluyeron 38 por duplicidad de la información y 20 por mediciones de calidad de vida con escalas diferentes al DLQI ${ }^{22,23,26-69}$.En la revisión de Basra, et al., se identificaron 44 artículos potenciales, de los cuales se excluyeron 34 por evaluar la calidad de vida antes y después de una intervención terapéutica o por utilizar escalas diferentes e idiomas diferentes al inglés ${ }^{56,60,64-96}$ Se incluyeron ocho artículos en el análisis final (FIGURA 1).

Los estudios incluidos en la revisión fueron observacionales. Sampogna ${ }^{103}$ agrupó los pacientes en hospitalizados menores de 65 años y de 65 o más años, y observó mayor deterioro en la calidad de vida en los últimos (DLQI: 8,8 Vs. 9,6). En contraste, Jager ${ }^{104}$ evaluó pacientes ambulatorios menores de 18 años y de 180 más años, y encontró un deterioro moderado en la calidad de vida similar en ambos grupos (DLQI: 6,8 $\pm 5,7$ ).

Meyer ${ }^{105}$ evaluó pacientes con psoriasis leve y moderada, y encontró en ambos tipos de pacientes deterioro moderado de su calidad de vida; la mayor proporción eran hombres $(51,3 \%)$, con una edad promedio de $58,8 \pm 13,8$ años. No se reportó la edad de inicio de la enfermedad ni su duración.

Los estudios de Schmitt ${ }^{106}$, Augustin ${ }^{107,108}$ y Meeuwis ${ }^{109}$ se hicieron en pacientes ambulatorios, y reportaron deterioro moderado; asimismo, la edad de inicio de la psoriasis estuvo a los $26,5 \pm 15,6$ años y el deterioro de 


\begin{tabular}{|c|c|c|c|c|c|c|c|c|c|}
\hline Augustin (107) & $2008 \Omega$ & Alemania & 1.511 & $50,5(14,9)$ & 57,8 & $17,6(14,5)$ & $33,5(17,6)$ & 8,6 & 12,0 \\
\hline Meeuwis (109) & 2011 & & 487 & $53,9(12,3)$ & 57,1 & - & $24,5(14,0)$ & $6,6(5,5)$ & - \\
\hline \multirow[t]{2}{*}{ Sampogna (103) } & 2005 & Italia & 792 & $<65$ & 59,3 & - & - & 8,8 & - \\
\hline & & & 144 & $\geq 65$ & 59 & - & - & 9,6 & - \\
\hline Lundberg (110) & 2000 & Suecia & 154 & $49,68(13,87)$ & 53 & $17,44(13,18)$ & & $4,98(4,94)$ & \\
\hline Augustin (108) & 2008 & Alemania & 2.009 & $51,5(14,6)$ & 56,3 & - & $21,3(15,2)$ & 7,5 & 10,1 \\
\hline
\end{tabular}

${ }^{\pi} \mathrm{DE}$ : desviación estándar; ${ }^{\circ} \mathrm{PASI}$ Score (Psoriasis Assessment and Severity Index)

La gravedad de la psoriasis según el PASI es: leve <7, moderada 7-12, grave $>12$.

^Dermatology Life Quality Index (DLQI); i pacientes con psoriasis grave; ii pacientes con psoriasis leve; ${ }^{\Omega}$ Augustin, fuente de los datos corresponde al 2005.

TABLA 1. Resumen estructurado de los ochos artículos con mejor nivel de evidencia sobre el impacto de la psoriasis en la calidad de vida, según variables demográficas y clínicas

la calidad de vida fue moderado. Sólo en el estudio de Lundberg ${ }^{110}$ se encontraron pacientes con una alteración leve en su calidad de vida. En cuanto al PASI, este sólo se reportó en los estudios de Augustin ${ }^{107,108}$ en pacientes que tenían enfermedad leve o moderada.

Sólo en tres estudios ${ }^{104,107,10}$ se reportó el tiempo de duración de la enfermedad, que estuvo entre 17,6 y 28,9 años.

De los artículos incluidos, se presenta la medida explícita publicada en cada estudio (TABLA 1).

\section{Discusión}

El DLQI es un cuestionario sencillo, de diez preguntas y fácil de diligenciar, por lo cual es muy útil para la práctica clínica rutinaria, ya que puede aplicarse a cualquier enfermedad dermatológica y permite comparar el impacto que cada una tiene sobre la calidad de vida de los pacientes; además, es el más empleado en pacientes con psoriasis ${ }^{17,19}$.

Otros cuestionarios para medir la calidad de vida en pacientes con lesiones dermatológicas, son Dermatology Quality of Life Scales (DQOLS) ${ }^{12}$, German Instrument for the Assessment of Quality of Life in Skin Diseases (DIELH) ${ }^{13,111}$, Dermatology Specific Quality of Life (DSQL) ${ }^{12}$ y algunos específicos para psoriasis tales como Psoriasis Disability Index ${ }^{133,114}$, Psoriasis Life Stress Inventory (PLSI) ${ }^{16}$, The Salford Psoriasis Index (SPI ${ }^{8} \mathrm{y}$ The Psoriasis Index of Quality of Life (PSORIQoL)', los cuales fueron objeto de revisión por Lewis, et al. ${ }^{7}$. Los resultados sobre cuál instrumento es el más idóneo depende del contexto evaluado, ya que hay variaciones en el tiempo de la historia natural de la enfermedad que mide cada instrumento, así como en aspectos psicométricos ${ }^{115,116}$. Por esta razón, no es posible comparar los resultados obtenidos entre estudios, pues habría un sesgo de información relacionado con el instrumento, porque cada uno de ellos evalúa aspectos diferentes de la calidad de vida.

Esta revisión sistemática basó sus resultados en el DLQI porque es el instrumento más utilizado en la práctica clínica y facilita la interpretación de la calidad de vida relacionada con la enfermedad en pacientes con psoriasis. Asimismo, tiene una gran validez externa porque permite comparar resultados medidos con el mismo instrumento en diferentes momentos de la historia natural de la enfermedad, sin que esto implique que este instrumento sea la mejor alternativa de medición de la calidad de vida en psoriasis, pero sí ayuda mucho a reducir la heterogeneidad de los resultados que se presenta al utilizar diferentes instrumentos de medición. 
Una debilidad de esta revisión sistemática es el bajo número de estudios que finalmente fueron incluidos, lo cual podría afectar la validez externa de sus resultados; aunque al mismo tiempo se gana validez interna por el gran control del sesgo de información, al considerar el mismo instrumento de medición de la calidad de vida en todos los estudios. La amplia utilización y la sencillez en el diligenciamiento del DLQI lo convierten en una importante opción para evaluar la calidad de vida en pacientes con psoriasis.

Otra debilidad de esta revisión es que no se presentan los resultados del DLQI de forma desagregada en los seis componentes; esto se debe a que en ninguno de los estudios incluidos se reportaron. Se recomienda que las investigaciones futuras presenten estos resultados desagregados para poder identificar el comportamiento de cada dominio de forma individual.

Aunque en esta revisión se evidenció una pérdida de la calidad de vida en los pacientes con psoriasis, no fue posible determinar la magnitud de dichos cambios en el tiempo, ya que todos los estudios incluidos fueron transversales. Esta afectación fue moderada en todos los estudios, excepto en el de Lundberg ${ }^{110}$. La gravedad de la enfermedad sólo se reportó en dos de los estudios $^{107,108}$, en los cuales los pacientes presentaban psoriasis moderada, por lo que se desconoce si dicha gravedad podría afectar la calidad de vida.

Seis de los ocho artículos incluidos (75\%) en esta revisión tuvieron una mayor proporción de pacientes de sexo masculino con psoriasis; asimismo, la enfermedad fue de larga evolución ( $>17$ años) y la psoriasis tuvo un inicio temprano (desde los 21 años), lo que según $\mathrm{Au}$ gustin, et al..$^{85}$, podría conducir a un mayor deterioro de la calidad de vida. La enfermedad de aparición temprana tiende a comportarse más agresivamente y suele acompañarse de un mayor número de enfermedades asociadas, por ejemplo, artritis psoriásica, lo cual alteraría más la calidad de vida de los pacientes.

La enfermedad afectó la calidad de vida en todos los casos; el PASI sólo fue reportado en dos de los artículos y se evidenció que las formas moderadas a graves de la enfermedad afectan la calidad de vida de los pacientes ${ }^{114}$.

En la revisión de Basra, et al. ${ }^{25}$, se observó que los pacientes con psoriasis tenían afectada su calidad de vida de forma moderada (DLQI promedio: 10,53), superior al resultado observado en pacientes con acné vulgar (DLQI: 7,45), alopecia (DLQI: 8,30), vitiligo (DLQI: 9,11) o urticaria (DLQI: 9,80).

Es importante poder determinar un índice de calidad de vida en todos los pacientes con psoriasis, independientemente de su gravedad, como el propuesto por Radke ${ }^{69}$. Al tener en cuenta la cronicidad de la enfermedad, se podría guiar el tratamiento con tratamientos farmacológicos dirigidos a mejorar la calidad de vida. Además, la gravedad de la enfermedad no es directamente proporcional al DQLI, es decir, hay pacientes gravemente afectados en piel y con un bajo valor en el DQLI, y viceversa. Cada paciente siente su enfermedad de manera diferente; por eso es tan importante no sólo hacer una medición inicial sino hacer un seguimiento continuo, con el fin de poder detectar tendencias en el cambio del DQLI y así intervenir oportunamente el tratamiento.

El DQLI es un instrumento de medición estandarizado para el médico tratante, que tiene un peso importante en la toma de decisiones terapéuticas. Aunque el paciente muestre formas leves de la enfermedad en piel pero valores de DQLI altos, es importante optar por medidas terapéuticas más agresivas, con el fin de mejorar la calidad de vida.

Características como las variables sociodemográficas de los pacientes, la duración de la enfermedad, la presencia de enfermedades dermatológicas concomitantes o enfermedades sistémicas, el tipo de psoriasis (vulgar, artritis psoriásica), la localización de las lesiones, la duración de la enfermedad, su gravedad, el uso concomitante de otros medicamentos (locales o sistémicos), pueden influir en los resultados de la calidad de vida de los pacientes con psoriasis, por lo cual es importante que se puedan hacer mediciones ajustadas por estas potenciales variables de confusión.

Es importante motivar al médico tratante a evaluar la calidad de vida de los pacientes con psoriasis, bien sea con el DLQI o con alguna otra escala de medición. Asimismo, el enfoque terapéutico en la psoriasis no debe depender sólo del tamaño de la superficie de piel afectada, ya que al igual que en otras enfermedades crónicas, también debe considerarse la calidad de vida, y mientras más oportuna sea la medición, más pronto podría obtenerse una mejoría clínica. El tratamiento proporciona alivio a estos pacientes, el cual mejora su calidad de vida, tal y como se ha evidenciado en diferentes ensayos clínicos que han hecho tales mediciones antes y después del tratamiento ${ }^{26,31,35,42,81,90}$.

Cada vez se hace más necesario un abordaje multidisciplinario del paciente con psoriasis, en el cual el psiquiatra y el psicólogo, por medio de terapias individuales o grupales, aborden los aspectos emocionales del paciente, ya que estos tienen un impacto muy importante sobre la evolución de la enfermedad. La intervención oportuna y temprana del plano psíquico del paciente, evitaría recaídas frecuentes de la enfermedad, así como el desarrollo de formas clínicas más graves. 


\section{Conclusión}

La psoriasis afecta la calidad de vida de los pacientes que padecen la enfermedad, tanto en sus formas leve y moderada, y este deterioro ha sido superior al encontrado en otras enfermedades dermatológicas como acné vulgar, vitiligo, alopecia y urticaria.

\section{Referencias}

1. Christophers E. Psoriasis -epidemiology and clinical spectrum. Clin Exp Dermatol. 2001;26:314-20.

2. Greaves MW, Weinstein GD. Treatment of psoriasis. N Engl J Med. 1995;332:581-8.

3. Krueger JG. The immunologic basis for the treatment of psoriasis with new biologic agents. J Am Acad Dermatol. 2002;46:1-23; quiz 23-26.

4. de Arruda LH, de Moraes AP. The impact of psoriasis on quality of life. Br J Dermatol. 2001;144(Suppl.58):33-6.

5. Krueger G, Koo J, Lebwohl M, Menter A, Stern RS, Rolstad T. The impact of psoriasis on quality of life: Results of a 1998 National Psoriasis Foundation patient-membership survey. Arch Dermatol. 2001;137:280-4.

6. Jenkinson C, Coulter A, Wright L. Short form 36 (SF36) health survey questionnaire: Normative data for adults of working age. BMJ. 1993;306:1437-40.

7. Lewis VJ, Finlay AY. A critical review of quality-of-life scales for psoriasis. Dermatol Clin. 2005;23:707-16.

8. Kirby B, Fortune DG, Bhushan M, Chalmers RJ, Griffiths CE. The Salford Psoriasis Index: An holistic measure of psoriasis severity. Br J Dermatol. 2000;142:728-32.

9. McKenna SP, Cook SA, Whalley D, Doward LC, Richards HL, Griffiths CEM, et al. Development of the PSORIQoL, a psoriasisspecific measure of quality of life designed for use in clinical practice and trials. Br J Dermatol. 2003;149:323-31.

10. Chren MM, Lasek RJ, Quinn LM, Mostow EN, Zyzanski SJ. Skindex, a quality-of-life measure for patients with skin disease: Reliability, validity, and responsiveness. J Invest Dermatol. 1996;107:707-13.

11. Chren MM, Lasek RJ, Sahay AP, Sands LP. Measurement properties of Skindex-16: A brief quality-of-life measure for patients with skin diseases. J Cutan Med Surg. 2001;5:105-10.

12. Morgan M, McCreedy R, Simpson J, Hay RJ. Dermatology quality of life scales -a measure of the impact of skin diseases. Br J Dermatol. 1997;136:202-6.

13. Schäfer T, Staudt A, Ring J. Development of the German Scale for Assessing Quality of Life in Skin Diseases. Hautarzt. 2001;52:492-8.

14. Chren MM, Lasek RJ, Flocke SA, Zyzanski SJ. Improved discriminative and evaluative capability of a refined version of Skindex, a quality-of-life instrument for patients with skin diseases. Arch Dermatol. 1997;133:1433-40.

15. Finlay AY, Kelly SE. Psoriasis -an index of disability. Clin Exp Dermatol. 1987;12:8-11.

16. Gupta MA, Gupta AK. The Psoriasis Life Stress Inventory: A preliminary index of psoriasis-related stress. Acta Derm Venereol. 1995;75:240-3.
17. Finlay AY, Khan GK. Dermatology Life Quality Index (DLQI) -a simple practical measure for routine clinical use. Clin Exp Dermatol. 1994;19:210-6.

18. Lewis V, Finlay AY. 10 years experience of the Dermatology Life Quality Index (DLQI). J Investig Dermatol Symp Proc. 2004;9:169-80.

19. Lewis VJ, Finlay AY. Two decades experience of the Psoriasis Disability Index. Dermatology (Basel). 2005;210:261-8.

20. Overview and Links | Dermatology Life Quality Index (DLQI). Fecha de consulta: 8 de febrero de 2012. Disponible en: http:// www.dermatology.org.uk/quality/dlqi/quality-dlqi.html.

21. Jankovic S, Raznatovic M, Marinkovic J, Jankovic J, Kocev N, Tomic-Spiric V, et al. Health-related quality of life in patients with psoriasis. J Cutan Med Surg. 2011;15:29-36.

22. Rakhesh SV, D’Souza M, Sahai A. Quality of life in psoriasis: A study from south India. Indian J Dermatol Venereol Leprol. 2008;74:600-6.

23. Sampogna F, Tabolli S, Söderfeldt B, Axtelius B, Aparo U, Abeni D. Measuring quality of life of patients with different clinical types of psoriasis using the SF-36. Br J Dermatol. 2006;154:844-9.

24. Abolfotouh MA, Al-Khowailed MS, Suliman WE, Al-Turaif DA, Al-Bluwi E, Al-Kahtani HS. Quality of life in patients with skin diseases in central Saudi Arabia. Int J Gen Med. 2012;5:633-42.

25. 25. Basra MKA, Fenech R, Gatt RM, Salek MS, Finlay AY. The Dermatology Life Quality Index 1994-2007: A comprehensive review of validation data and clinical results. Br J Dermatol. 2008;159:997-1035.

26. 26. Tyring S, Gottlieb A, Papp K, Gordon K, Leonardi C, Wang A, et al. Etanercept and clinical outcomes, fatigue, and depression in psoriasis: Double-blind placebo-controlled randomized phase III trial. Lancet. 2006;367:29-35.

27. Asawanonda P, Chingchai A, Torranin P. Targeted UV-B phototherapy for plaque-type psoriasis. Arch Dermatol. 2005;141:1542-6.

28. Ortonne J-P, Shear N, Shumack S, Henninger E. Impact of efalizumab on patient-reported outcomes in high-need psoriasis patients: Results of the international, randomized, placebocontrolled Phase III Clinical Experience Acquired with Raptiva (CLEAR) trial [NCTo0256139]. BMC Dermatol. 2005;5:13.

29. Krueger GG, Langley RG, Finlay AY, Griffiths CEM, Woolley JM, Lalla D, et al. Patient-reported outcomes of psoriasis improvement with etanercept therapy: Results of a randomized phase III trial. Br J Dermatol. 2005;153:1192-9.

30. Prins M, Krabbe PFM, Swinkels QOJ, De Boo T, van de Kerkhof PCM, van der Valk PGM. The effect of treatment on quality of life in psoriasis patients. Acta Derm Venereol. 2005;85:304-10.

31. Feldman SR, Gordon KB, Bala M, Evans R, Li S, Dooley LT, et al. Infliximab treatment results in significant improvement in the quality of life of patients with severe psoriasis: A double-blind placebo-controlled trial. Br J Dermatol. 2005;152:954-60.

32. Menter A, Gordon K, Carey W, Hamilton T, Glazer S, Caro I, et al. Efficacy and safety observed during 24 weeks of efalizumab therapy in patients with moderate to severe plaque psoriasis. Arch Dermatol. 2005;141:31-8.

33. Ricardo RR, Rhoa M, Orenberg EK, Li N, Rundle AC, Caro I. Clinical benefits in patients with psoriasis after efalizumab therapy: Clinical trials versus practice. Cutis. 2004;74:193-200.

34. Gottlieb AB, Evans R, Li S, Dooley LT, Guzzo CA, Baker D, et al. Infliximab induction therapy for patients with severe plaque-type psoriasis: A randomized, double-blind, placebo-controlled trial. J Am Acad Dermatol. 2004;51:534-42. 
35. van de Kerkhof PCM. The impact of a two-compound product containing calcipotriol and betamethasone dipropionate (Daivobet/ Dovobet) on the quality of life in patients with psoriasis vulgaris: A randomized controlled trial. Br J Dermatol. 2004;151:663-8.

36. Opmeer BC, Heydendael VMR, De Borgie CAJM, Spuls PI, Bossuyt PMM, Bos JD, et al. Costs of treatment in patients with moderate to severe plaque psoriasis: Economic analysis in a randomized controlled comparison of methotrexate and cyclosporine. Arch Dermatol. 2004;140:685-90.

37. Fortune DG, Richards HL, Griffiths CEM, Main CJ. Targeting cognitive-behaviour therapy to patients' implicit model of psoriasis: Results from a patient preference controlled trial. Br J Clin Psychol. 2004;43:65-82.

38. Feldman SR, Menter A, Koo JY. Improved health-related quality of life following a randomized controlled trial of alefacept treatment in patients with chronic plaque psoriasis. $\mathrm{Br} J$ Dermatol. 2004;150:317-26.

39. Gordon KB, Papp KA, Hamilton TK, Walicke PA, Dummer W, Li $\mathrm{N}$, et al. Efalizumab for patients with moderate to severe plaque psoriasis: A randomized controlled trial. JAMA. 2003;290:3073-80.

40. Gottlieb AB, Matheson RT, Lowe N, Krueger GG, Kang S, Goffe BS, et al. A randomized trial of etanercept as monotherapy for psoriasis. Arch Dermatol. 2003;139:1627-1632; discussion 1632.

41. Gordon KB, Vaishnaw AK, O’Gorman J, Haney J, Menter A. Treatment of psoriasis with alefacept: Correlation of clinical improvement with reductions of memory T-cell counts. Arch Dermatol. 2003;139:1563-70.

42. Bergstrom KG, Arambula K, Kimball AB. Medication formulation affects quality of life: A randomized single-blind study of clobetasol propionate foam $0.05 \%$ compared with a combined program of clobetasol cream $0.05 \%$ and solution $0.05 \%$ for the treatment of psoriasis. Cutis. 2003;72:407-11.

43. Leonardi CL, Powers JL, Matheson RT, Goffe BS, Zitnik R, Wang A, et al. Etanercept as monotherapy in patients with psoriasis. $\mathrm{N}$ Engl J Med. 2003;349:2014-22.

44. Heydendael VMR, Spuls PI, Opmeer BC, De Borgie CAJM, Reitsma JB, Goldschmidt WFM, et al. Methotrexate versus cyclosporine in moderate-to-severe chronic plaque psoriasis. $\mathrm{N}$ Engl J Med. 2003:349:658-65.

45. Woo WK, McKenna KE. Combination TLo1 ultraviolet B phototherapy and topical calcipotriol for psoriasis: A prospective randomized placebo-controlled clinical trial. Br J Dermatol. 2003;149:146-50.

46. Housman TS, Keil KA, Mellen BG, McCarty MA, Fleischer AB Jr, Feldman SR. The use of $0.25 \%$ zinc pyrithione spray does not enhance the efficacy of clobetasol propionate $0.05 \%$ foam in the treatment of psoriasis. J Am Acad Dermatol. 2003;49:79-82.

47. Schiffner R, Schiffner-Rohe J, Gerstenhauer M, Hofstädter F, Landthaler M, Stolz W. Willingness to pay and time trade-off: Sensitive to changes of quality of life in psoriasis patients? $\mathrm{Br} \mathrm{J}$ Dermatol. j 2003;148:1153-60.

48. Ortonne J-P. Clinical response to alefacept: Results of a phase 3 study of intramuscular administration of alefacept in patients with chronic plaque psoriasis. J Eur Acad Dermatol Venereol. 2003;17(Suppl.2):12-6.

49. Finlay AY, Salek MS, Haney J. Intramuscular alefacept improves health-related quality of life in patients with chronic plaque psoriasis. Dermatology (Basel). 2003;206:307-15.
50. Ellis CN, Mordin MM, Adler EY. Effects of alefacept on healthrelated quality of life in patients with psoriasis: Results from a randomized, placebo-controlled phase II trial. Am J Clin Dermatol. 2003;4:131-9.

51. Gradwell C, Thomas KS, English JSC, Williams HC. A randomized controlled trial of nurse follow-up clinics: Do they help patients and do they free up consultants' time? Br J Dermatol. 2002;147:513-7.

52. Fortune DG, Richards HL, Kirby B, Bowcock S, Main CJ, Griffiths CEM. A cognitive-behavioural symptom management programme as an adjunct in psoriasis therapy. Br J Dermatol. 2002;146:458-65.

53. Touw CR, Hakkaart-van Roijen L, Verboom P, Paul C, Rutten FF, Finlay AY. Quality of life and clinical outcome in psoriasis patients using intermittent cyclosporin. Br J Dermatol. 2001;144:967-72.

54. James M. A randomized, double-blind, multicenter trial comparing fluticasone propionate cream, $0.05 \%$, and hydrocortisone17-butyrate cream, $0.1 \%$, applied twice daily for 4 weeks in the treatment of psoriasis. Cutis. 2001;67(Suppl.):2-9.

55. Ho VC, Griffiths CE, Berth-Jones J, Papp KA, Vanaclocha F, Dauden $\mathrm{E}$, et al. Intermittent short courses of cyclosporine microemulsion for the long-term management of psoriasis: A 2-year cohort study. J Am Acad Dermatol. 2001;44:643-51.

56. Leonardi CL. Efalizumab: An overview. J Am Acad Dermatol. 2003;49(Suppl.):S98-104

57. Poulin Y, Papp KA, Wasel NR, Andrew R, Fraquelli E, Bernstein $\mathrm{G}$, et al. A Canadian online survey to evaluate awareness and treatment satisfaction in individuals with moderate to severe plaque psoriasis. Int J Dermatol. 2010;49:1368-75.

58. Aghaei S, Moradi A, Ardekani GS. Impact of psoriasis on quality of life in Iran. Indian J Dermatol Venereol Leprol. 2009;75:220.

59. Zachariae R, Zachariae C, Ibsen H, Mortensen JT, Wulf HC. Dermatology life quality index: Data from Danish inpatients and outpatients. Acta Derm Venereol. 2000;80:272-6.

6o. Mazzotti E, Barbaranelli C, Picardi A, Abeni D, Pasquini P. Psychometric properties of the Dermatology Life Quality Index (DLQI) in 900 Italian patients with psoriasis. Acta Derm Venereol. 2005;85:409-13.

61. Colombo G, Altomare G, Peris K, Martini P, Quarta G, Congedo M, et al. Moderate and severe plaque psoriasis: Cost-of-illness study in Italy. Ther Clin Risk Manag. 2008;4:559-68.

62. Reich K, Krüger K, Mössner R, Augustin M. Epidemiology and clinical pattern of psoriatic arthritis in Germany: A prospective interdisciplinary epidemiological study of 1511 patients with plaque-type psoriasis. Br J Dermatol. 2009;160:1040-7.

63. Chernyshov PV, Bylatte M. Comparative study of quality of life in patients with psoriasis from Lithuania and Ukraine. Lik Sprava. 2010; 7-8:107-11.

64. Sato R, Milligan G, Molta C, Singh A. Health-related quality of life and healthcare resource use in European patients with plaque psoriasis: An association independent of observed disease severity. Clin Exp Dermatol. 2011;36:24-8.

65. Bilac C, Ermertcan AT, Bilac DB, Deveci A, Horasan GD. The relationship between symptoms and patient characteristics among psoriasis patients. Indian J Dermatol Venereol Leprol. 2009;75:551.

66. Wahl AK, Mørk C, Lillehol BM, Myrdal AM, Helland S, Hanestad $\mathrm{BR}$, et al. Changes in quality of life in persons with eczema and psoriasis after treatment in departments of dermatology. Acta Derm Venereol. 2006;86:198-201. 
67. Mazzotti E, Picardi A, Sampogna F, Sera F, Pasquini P, Abeni D. Sensitivity of the Dermatology Life Quality Index to clinical change in patients with psoriasis. Br J Dermatol. 2003;149:318-22.

68. Lin T-Y, See L-C, Shen Y-M, Liang C-Y, Chang H-N, Lin Y-K. Quality of life in patients with psoriasis in northern Taiwan. Chang Gung Med J. 2011;34:186-96.

69. Radtke MA, Reich K, Blome C, Kopp I, Rustenbach SJ, Schäfer $\mathrm{I}$, et al. Evaluation of quality of care and guideline-compliant treatment in psoriasis. Development of a new system of quality indicators. Dermatology (Basel). 2009;219:54-8.

70. Nijsten T, Meads DM, De Korte J, Sampogna F, Gelfand JM, Ongenae $\mathrm{K}$, et al. Cross-cultural inequivalence of dermatologyspecific health-related quality of life instruments in psoriasis patients. J Invest Dermatol. 2007;127:2315-22.

71. Badia X, Mascaró JM, Lozano R. Measuring health-related quality of life in patients with mild to moderate eczema and psoriasis: Clinical validity, reliability and sensitivity to change of the DLQI. The Cavide Research Group. Br J Dermatol. 1999;141:698-702.

72. Shikiar R, Bresnahan BW, Stone SP, Thompson C, Koo J, Revicki DA. Validity and reliability of patient reported outcomes used in psoriasis: Results from two randomized clinical trials. Health Qual Life Outcomes. 2003;1:53.

73. Shikiar R, Willian MK, Okun MM, Thompson CS, Revicki DA. The validity and responsiveness of three quality of life measures in the assessment of psoriasis patients: Results of a phase II study. Health Qual Life Outcomes. 2006;4:71.

74. Mørk C, Wahl A. Improved quality of life among patients with psoriasis after supervised climate therapy at the Canary Islands. J Am Acad Dermatol. 2002;47:314-6.

75. Menter A, Kosinski M, Bresnahan BW, Papp KA, Ware JE Jr. Impact of efalizumab on psoriasis-specific patient-reported outcomes. Results from three randomized, placebo-controlled clinical trials of moderate to severe plaque psoriasis. J Drugs Dermatol. 2004;3:27-38.

76. Finlay AY. Current severe psoriasis and the rule of tens. Br J Dermatol. 2005;152:861-7.

77. Nichol MB, Margolies JE, Lippa E, Rowe M, Quell J. The application of multiple quality-of-life instruments in individuals with mild-tomoderate psoriasis. Pharmacoeconomics. 1996;10:644-53.

78. Sampogna F, Sera F, Abeni D. Measures of clinical severity, quality of life, and psychological distress in patients with psoriasis: A cluster analysis. J Invest Dermatol. 2004;122:602-7.

79. McKenna SP, Lebwohl M, Kahler KN. Development of the US PSORIQoL: A psoriasis-specific measure of quality of life. Int J Dermatol. 2005;44:462-9.

8o. Schmitt JM, Ford DE. Role of depression in quality of life for patients with psoriasis. Dermatology (Basel). 2007;215:17-27.

81. Schmitt J, Heese E, Wozel G, Meurer M. Effectiveness of inpatient treatment on quality of life and clinical disease severity in atopic dermatitis and psoriasis vulgaris -a prospective study. Dermatology (Basel). 2007;214:68-76.

82. Schmid-Ott G, Burchard R, Niederauer HH, Lamprecht F, Künsebeck H-W. Stigmatization and quality of life of patients with psoriasis and atopic dermatitis. Hautarzt. 2003;54:852-7.

83. Heinen-Kammerer T, Daniel D, Stratmann L, Rychlik R, Boehncke $\mathrm{W}-\mathrm{H}$. Cost-effectiveness of psoriasis therapy with etanercept in Germany. J Dtsch Dermatol Ges. 2007;5:762-8.

84. Schöffski O, Augustin M, Prinz J, Rauner K, Schubert E, Sohn S, et al. Costs and quality of life in patients with moderate to severe plaque-type psoriasis in Germany: A multi-center study. J Dtsch Dermatol Ges. 2007;5:209-18.
85. Augustin M, Zschocke I, Lange S, Seidenglanz K, Amon U. Quality of life in skin diseases: Methodological and practical comparison of different quality of life questionnaires in psoriasis and atopic dermatitis. Hautarzt. 1999;50:715-22.

86. Antoniou C, Stefanaki I, Stratigos A, Avgerinou G, Stavropoulos $\mathrm{P}$, Potouridou I, et al. The Greek experience with efalizumab in psoriasis from a University Dermatologic Hospital. Br J Dermatol. 2007;156(Suppl.2):12-6.

87. Sampogna F, Gisondi P, Tabolli S, Abeni D. Impairment of sexual life in patients with psoriasis. Dermatology (Basel). 2007;214:144-50.

88. De Felice C, Mazzotta A, Esposito M, Bianchi L, Chimenti S. Highdose initiation of etanercept in psoriatic arthritis and plaque psoriasis: Efficacy, safety and impact on patients' quality of life. J Dermatolog Treat. 2006;17:355-8.

89. Papoutsaki M, Chimenti M-S, Costanzo A, Talamonti M, Zangrilli A, Giunta A, et al. Adalimumab for severe psoriasis and psoriatic arthritis: An open-label study in 30 patients previously treated with other biologics. J Am Acad Dermatol. 2007;57:269-75.

90. Asawanonda P, Nateetongrungsak Y. Methotrexate plus narrowband UVB phototherapy versus narrowband UVB phototherapy alone in the treatment of plaque-type psoriasis: A randomized, placebo-controlled study. J Am Acad Dermatol. 2006;54:1013-8.

91. Woolacott N, Hawkins N, Mason A, Kainth A, Khadjesari Z, Vergel $\mathrm{YB}$, et al. Etanercept and efalizumab for the treatment of psoriasis: A systematic review. Health Technol Assess. 2006;10:1-233, i-iv.

92. Salim A, Tan E, Ilchyshyn A, Berth-Jones J. Folic acid supplementation during treatment of psoriasis with methotrexate: A randomized, double-blind, placebo-controlled trial. Br J Dermatol. 2006;154:1169-74.

93. Smith $\mathrm{CH}$, Jackson K, Bashir SJ, Pérez A, Chew AL, Powell AM, et al. Infliximab for severe, treatment-resistant psoriasis: A prospective, open-label study. Br J Dermatol. 2006;155:160-9.

94. Vensel E, Hilley T, Trent J, Taylor JR, Kirsner RS, Kerdel FA, et al. Sustained improvement of the quality of life of patients with psoriasis after hospitalization. J Am Acad Dermatol. 2000;43:858-60.

95. Gottlieb AB, Mease PJ, Mark Jackson J ¿?, Eisen D, Amy Xia H, Asare $\mathrm{C}$, et al. Clinical characteristics of psoriatic arthritis and psoriasis in dermatologists' offices. J Dermatolog Treat. 2006;17:279-87.

96. Katugampola RP, Hongbo Y, Finlay AY. Clinical management decisions are related to the impact of psoriasis on patient-rated quality of life. Br J Dermatol. 2005;152:1256-62.

97. Wahl AK, Mørk C, Hanestad BR, Helland S. Coping with exacerbation in psoriasis and eczema prior to admission in a dermatological ward. Eur J Dermatol. 2006;16:271-5.

98. Uttjek M, Dufåker M, Nygren L, Stenberg B. Determinants of quality of life in a psoriasis population in northern Sweden. Acta Derm Venereol. 2004;84:37-43.

99. Zaghloul SS, Goodfield MJD. Objective assessment of compliance with psoriasis treatment. Arch Dermatol. 2004;140:408-14.

100. Eghlileb AM, Davies EEG, Finlay AY. Psoriasis has a major secondary impact on the lives of family members and partners. Br J Dermatol. 2007;156:1245-50.

101. Türel Ermertcan A, Temelta G, Deveci A, Dinç G, Güler HB, Oztürkcan S. Sexual dysfunction in patients with psoriasis. J Dermatol. 2006;33:772-8.

102. Al-Mazeedi K, El-Shazly M, Al-Ajmi HS. Impact of psoriasis on quality of life in Kuwait. Int J Dermatol. 2006;45:418-24. 
103. Sampogna F, Chren MM, Melchi CF, Pasquini P, Tabolli S, Abeni D. Age, gender, quality of life and psychological distress in patients hospitalized with psoriasis. Br J Dermatol. 2006;154:325-31.

104. De Jager MEA, De Jong EMGJ, van der Kerkhof PCM, Evers AWM, Seyger MMB. An intrapatient comparison of quality of life in psoriasis in childhood and adulthood. J Eur Acad Dermatol Venereol. 2011;25:828-31.

105. Meyer N, Paul C, Feneron D, Bardoulat I, Thiriet C, Camara C, et al. Psoriasis: An epidemiological evaluation of disease burden in 590 patients. J Eur Acad Dermatol Venereol. 2010;24:1075-82.

106. Schmitt JM, Ford DE. Work limitations and productivity loss are associated with health-related quality of life but not with clinical severity in patients with psoriasis. Dermatology (Basel). 2006;213:102-10.

107. Augustin M, Krüger K, Radtke MA, Schwippl I, Reich K. Disease severity, quality of life and health care in plaque-type psoriasis: A multicenter cross-sectional study in Germany. Dermatology (Basel). 2008;216:366-72.

108. Augustin M, Reich K, Reich C, Purwins S, J Rustenbach J, Schäfer $\mathrm{I}$, et al. Quality of psoriasis care in Germany -results of the national study PsoHealth 2007. J Dtsch Dermatol Ges. 2008;6:640-5.

109. Meeuwis KAP, De Hullu JA, van de Nieuwenhof HP, Evers AWM, Massuger LFAG, van de Kerkhof PCM, et al. Quality of life and sexual health in patients with genital psoriasis. Br J Dermatol. 2011;164:1247-55.
110. Lundberg L, Johannesson M, Silverdahl M, Hermansson C, Lindberg M. Health-related quality of life in patients with psoriasis and atopic dermatitis measured with SF-36, DLQI and a subjective measure of disease activity. Acta Derm Venereol. 2000;80:430-4.

111. Schäfer T, Staudt A, Ring J. German instrument for the assessment of quality of life in skin diseases (DIELH). Internal consistency, reliability, convergent and discriminant validity and responsiveness. Hautarzt. 2001;52:624-8.

112. Anderson RT, Rajagopalan R. Development and validation of a quality of life instrument for cutaneous diseases. J Am Acad Dermatol. 1997;37:41-50.

113. Finlay AY, Kelly SE. Psoriasis -an index of disability. Clin Exp Dermatol. 1987;12:8-11.

114. Finlay AY, Coles EC. The effect of severe psoriasis on the quality of life of 369 patients. Br J Dermatol. 1995;132:236-44.

115. Both H, Essink-Bot M-L, Busschbach J, Nijsten T. Critical review of generic and dermatology-specific health-related quality of life instruments. J Invest Dermatol. 2007;127:2726-39.

116. Bronsard V, Paul C, Prey S, Puzenat E, Gourraud P-A, Aractingi S, et al. What are the best outcome measures for assessing quality of life in plaque type psoriasis? A systematic review of the literature. J Eur Acad Dermatol Venereol. 2010;24(Suppl.2):17-22. 